Article

\title{
A Self-Consistent Quantum Field Theory for Random Lasing
}

\author{
Andreas Lubatsch ${ }^{1,2,+}$ (i) and Regine Frank ${ }^{1,3, *,+}$ \\ 1 Physikalisches Institut, Rheinische Friedrich-Wilhelms Universität Bonn, Wegelerstr. 8, 53115 Bonn, \\ Germany; lubatsch@th.physik.uni-bonn.de \\ 2 Georg-Simon-Ohm University of Applied Sciences, Keßlerplatz 12, 90489 Nürnberg, Germany \\ 3 Serin Physics Laboratory, Department of Physics and Astronomy, Rutgers University, 136 Frelinghuysen \\ Road, Piscataway, NJ 08854-8019, USA \\ * Correspondence: regine.frank@rutgers.edu or regine.frank@googlemail.com \\ + These authors contributed equally to this work.
}

Received: 4 April 2019; Accepted: 8 June 2019; Published: 18 June 2019

check for updates

\begin{abstract}
The spatial formation of coherent random laser modes in strongly scattering disordered random media is a central feature in the understanding of the physics of random lasers. We derive a quantum field theoretical method for random lasing in disordered samples of complex amplifying Mie resonators which is able to provide self-consistently and free of any fit parameter the full set of transport characteristics at and above the laser phase transition. The coherence length and the correlation volume respectively is derived as an experimentally measurable scale of the phase transition at the laser threshold. We find that the process of stimulated emission in extended disordered arrangements of active Mie resonators is ultimately connected to time-reversal symmetric multiple scattering in the sense of photonic transport while the diffusion coefficient is finite. A power law is found for the random laser mode diameters in stationary state with increasing pump intensity.
\end{abstract}

Keywords: multiple scattering; random laser; quantum field theory; Mie resonance

\section{Introduction}

The research for random lasers is an emerging research field [1-11], which recently has been extended to highly flexible [12] and unconventional materials and setups [13,14]. If these systems can be operated spectrally and spatially well controlled they feature the future as large area coherent light sources ahead of all state of the art LEDs. Theoretically many different models from statistical physics [15-22], classical field theoretical methods [23-27], quantum dynamical [28-30] and quantum field theoretical approaches [31-34] for embedded disordered ensembles of laser active scatterers $[35,36]$ are investigated. The random laser setup consists of a multiple scattering medium which can be also passive Mie scatterers infiltrated by laser active dye [37] and dye infiltrated disordered waveguides [38]. Random lasers are operated in absence of any external feedback or mirror system, thus it is of principal importance that a high contrast of the refractive index between scatterer and background is given in order to enhance multiple scattering and thus photonic transport and as a result of the accumulation of a high number of photons in the sample. A scheme of a possible setup for monodisperse solid state Mie scatterers is found in Figure 1a. Monodisperse Mie spheres can become in certain configurations extremely sensible systems, especially when the scatterers are large compared to the transport wavelength. In this regime the Mie spheres support the occurrence of intensity fluctuations. We chose a system of monodisperse scatterers for this article in order to check systematically whether the signature of the independent single Mie scatterer in the transport characteristics of the non-pumped and non-inverted microscopic system will persist in the stationary 
state of lasing. We develop a quantum field theoretical approach of photonic transport based on the Bethe-Salpeter equation, see Figure 1c, that incorporates all orders of interference effects by means of the Cooperon, the maximally crossed Feynman diagram [39], see Figure 1d. Energy conservation laws are implemented by means of a generalized Ward identity [40]. We couple this framework to the microscopic laser rate equations for quantum cascades, see Figure $1 \mathrm{~b}$, that ensure particle conservation on the microscopic level.
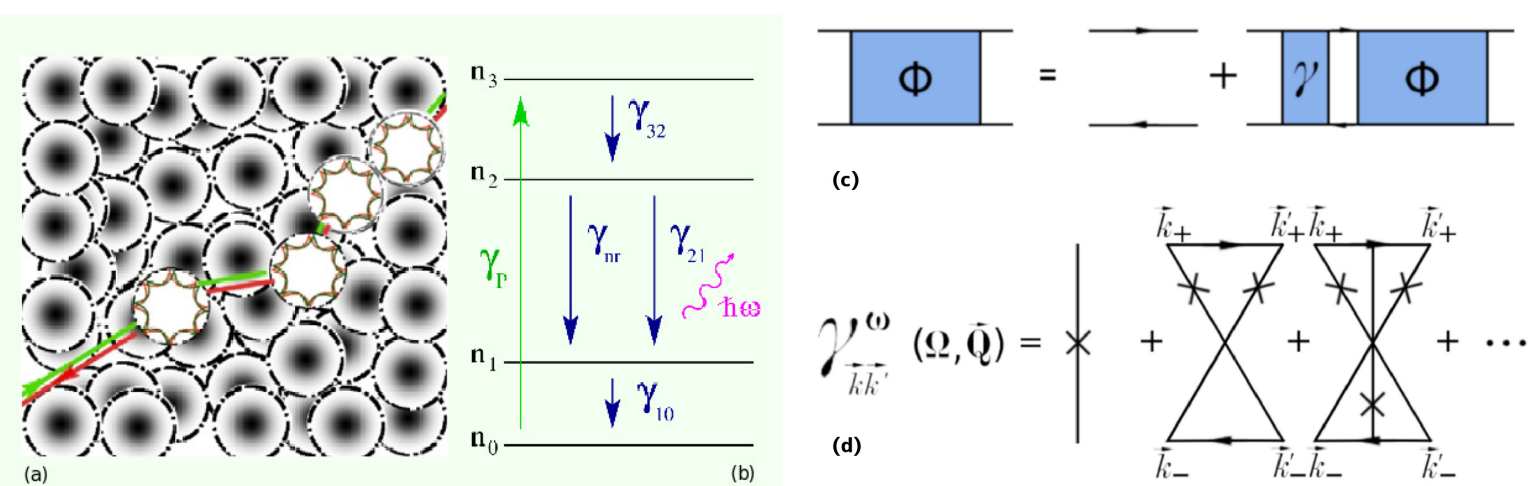

Figure 1. (a) Sketch of a disordered semi-conductor random laser slab, that is open in $z$-direction. Photonic transport processes (red) and their time reversal (green) interfere while they feature multiple scattering processes with complex active Mie resonators. The Mie resonance [41] is a so called whispering gallery resonance at the inner surface of the independent Mie scatterer. We use for this work monodisperse Mie spheres. (b) 4-level laser rate equation scheme. Straight lines represent the electronic processes, the green line represents the excitation due to the pump field, $\gamma_{32}$ represents the decay from level $n_{3}$ to the upper laser level $n_{2}, \gamma_{n r}$ represents nonradiative decay and as its competitor $\gamma_{21}$ leads to spontaneous and to stimulated emission processes, which eventually yields inversion and coherent laser radiation $\hbar \omega$. (c) Schematic representation of the Bethe-Salpeter equation, compare Equation (6). $\Phi$ is the intensity field in the sample, $\gamma_{\vec{k} k^{\prime}}^{\omega}$ is the irreducible vertex. (d) The irreducible vertex includes all orders of maximally crossed diagrams (Cooperon) which represent all quantum-coherent interference contributions due to multiple scattering in presence of disorder.

For three dimensional random lasers of finite and possibly large extent theoretical frameworks often come along with tremendous computational efforts. Our approach is one theoretical possibility to deal with dense ensembles of strongly scattering resonators efficiently, either in the case of independent scatterers or in the case of photonic glasses. We derive in the following a self-consistent frame which provides systematic results free of any fit parameter that are directly measurable in random laser experiments.

\section{Quantum-Field Theory for Photonic Transport}

We develop in this article a self-consistent quantum field theory for random lasing of ensembles of active Mie resonators. The results are spatial characteristics of the phase transition to random lasing, they classify the threshold to stationary state lasing. The theory includes multiple scattering effects of photons in complex random arrangements which may be classified in incoherent contributions that are expressed in quantum field theory, e.g., as ladder diagrams, the diffuson, and coherent contributions, that are expressed in terms of maximally crossed diagrams, the Cooperon. Methodologically the Bethe-Salpeter equation, see Figure 1c, is a most reasonable self-consistent frame, where the so called memory kernel $M$ as part of the irreducible vertex $\gamma$ bares the expansion in orders of maximally crossed diagrams, Figure 1d. All light-matter interactions are represented within the irreducible vertex, e.g., the interaction of the electromagnetic field and its time reversal as well as the light intensity with a complex Mie resonator as a scatterer [40]. Samples of a large number of active Mie resonators 
can be considered, which may act as independent scatterers or as glasses, i.e., as an accumulation of agglomerates with an intrinsic correlation. In what follows we derive this theory for the case of monodisperse independent active Mie scatterers, where pronounced dips were found theoretically and experimentally in the results for the transport characteristics, e.g., the scattering mean-free path $l_{s}$ [34].

\subsection{Nonlinear Response}

The underlying electrodynamics for transport in non-linear media is described basically by the wave equation for Kerr-Media

$$
\Delta \vec{E}-\frac{\epsilon}{c^{2}} \frac{\partial^{2} \vec{E}}{\partial t^{2}}=\frac{1}{c^{2} \epsilon_{0}} \frac{\partial^{2} \vec{P}}{\partial t^{2}}
$$

where the polarizability $\vec{P}(\vec{E})$ may be decomposed in linear and non-linear part $\vec{P}(\vec{E})=\epsilon_{0}(\epsilon-1) \vec{E}+$ $\vec{P}_{N L}$. The electric displacement inside a medium is written as $\vec{D}(\vec{E})=\epsilon_{0} \epsilon \vec{E}+\vec{P}_{N L}$. Kerr media are $\chi^{(2)}$ or higher order processes in their dependency to the electrical field $E$.

$$
\vec{P}=\epsilon_{0} \chi^{(1)} \vec{E}+\epsilon_{0} \chi^{(2)} \vec{E} \cdot \vec{E}+\ldots
$$

\subsection{Bethe-Salpeter Equation for Photonic Transport in Samples of Disordered Active Mie Resonators}

We consider the propagating photonic intensity, see Figure 1c, as the field field correlator $\Phi$ which is derived selfconsistently by the Bethe-Salpeter equation. The propagating electromagnetic wave in disordered random media is described by the single-particle Green's function Equation (3) that solves the non-linear wave equation, Equation (1).

$$
G_{\vec{k}}^{\omega}=\frac{1}{\epsilon_{b}(\omega / c)^{2}-|\vec{k}|^{2}-\Sigma_{\vec{k}}^{\omega}}
$$

The scatterers are embedded in a background matrix, with the dielectric function $\epsilon_{b}$, e.g., in air. The granularity of the random medium is implemented in the form of a spatially dependent potential of the permittivity $\epsilon_{s}(\vec{r})$. As a result of our theoretical framework we derive the laser gain self-consistently and space-resolved. The order of non-linearity is a matter of the numerical self-consistency as the independent complex semiconductor scatterers are well described by the T-matrix [40] for spherical Mie resonators of matter with a self-consistent complex refractive index.

We use a model of independent Mie scatterers, Figure 1b, here, in order to derive the self-energy contribution $\Sigma_{\vec{k}}^{\omega}$ for extended photo-active particles in Equation (3). The Mie scattering coefficients of n-th order are known from the literature [41] to have the following form

$$
\begin{aligned}
a_{n} & =\frac{m \Psi_{n}(m y) \Psi_{n}^{\prime}(y)-\Psi_{n}(y) \Psi_{n}^{\prime}(m y)}{m \Psi_{n}(m y) \xi_{n}^{\prime}(y)-\xi_{n}(y) \Psi_{n}^{\prime}(m y)} \\
b_{n} & =\frac{\Psi_{n}(m y) \Psi_{n}^{\prime}(y)-m \Psi_{n}(y) \Psi_{n}^{\prime}(m y)}{\Psi_{n}(m y) \xi_{n}^{\prime}(y)-m \xi_{n}(y) \Psi_{n}^{\prime}(m y) .}
\end{aligned}
$$

In this notation $m=\frac{n_{s}}{n_{b}}$ is the relative refractive index between the scatterer and the background matrix, $y=\frac{2 \pi n_{s} r_{S}}{\lambda}$ is the size parameter depending on the scatterers' radius $r_{S}$ as well as on the transport wavelength $\lambda . \Psi_{n}, \xi_{n}$ are Riccati-Bessel functions. The refractive index $n_{s}(\Phi)$ obtains a higher-order non-linearity as consequence of the self-consistency with respect to $\Phi, \operatorname{Im} \epsilon_{\mathcal{S}}(\Phi)$. The independent scatterer approach works very well in dense random media of filling of $35-55 \%$. Positional dependent enhancement effects due to the high filling fraction are effectively mapped on the dynamics of $n_{s}(\Phi)$ of the single scatterer in non-linear response, and we use the exact Mie scattering solution. 
The Green's function, Equation (3), for $\Psi_{\omega}$, solves the wave equation Equation (1) and builds up the intensity field in the sample in the form of the field correlator $\Phi_{\vec{k} \vec{k}^{\prime}}^{\omega}(\vec{Q}, \Omega)$. The four point correlator

$$
\Phi_{\vec{k} \vec{k}^{\prime}}^{\omega}(\vec{Q}, \Omega)=\left\langle\hat{G}_{\vec{k}_{+} \vec{k}_{+}^{\prime}}^{R}(\omega) \hat{G}_{\vec{k}_{-} \vec{k}_{-}^{\prime}}^{A}(\omega)\right\rangle
$$

is thus expressed in terms of retarded and advanced Green's function $\hat{G}^{R / A}$ denoting the field and its time-reversal. We utilize the transformation of coordinates in center-of-motion $(\vec{Q}, \Omega)$ and relative $(\vec{k}, \omega)$ momenta and frequencies [34] $\vec{k}_{ \pm}=\vec{k} \pm \vec{Q} / 2$ and $\omega_{ \pm}=\omega \pm \Omega / 2$. Considering the slab geometry to be extended within the $(x, y)$-plane and finite in $z$-direction, the full Fourier transform as indicated for infinite samples [31] is replaced by a partial Fourier transform following the argument of the separation of the scales for the field $\Psi$ and the intensity $\Phi$. $\Psi$ is characterized by the wavelength $\lambda$, whereas the change of the light intensity $\Phi$ is characterized by the transport mean-free path $\xi$, and the correlation length in random lasers respectively, which is one of the central characteristics that we derive in due course of the paper. In $(x, y)$-plane the standard Fourier transform is used, in the limited $z$-direction we Fourier-transform the relative coordinate but the center-of-motion coordinate $Z$ remains in real space. The separation of scales allows for the physical incorporation of loss at the samples boundaries at the level of the transport theory. The break of the T-symmetry, the break of the time reversal invariance of the multiple scattering processes, is incorporated in a macrocanonical sense [42] on the level of the integration of the real space coordinate $Z$. We apply these arguments to the equation of motion for the intensity correlation, known as the Bethe-Salpeter equation, see Figure 1c,

$$
\Phi_{\epsilon \epsilon}=G^{R} G^{A}\left[1+\int \frac{d^{3} k}{(2 \pi)^{3}} \gamma \Phi_{\epsilon \epsilon}\right]
$$

and we obtain the Boltzmann or kinetic equation for transport Equation (8). We introduce here the following abbreviations, consistent with previous work, $\Delta \Sigma=\Sigma^{A}\left(\omega_{-}\right)-\Sigma^{R}\left(\omega_{+}\right), \square \Sigma=$ $\Sigma^{A}\left(\omega_{-}\right)+\Sigma^{R}\left(\omega_{+}\right)$, and equivalent expressions for $\Delta G_{\vec{k}-}^{\omega_{-}}$and $\square G_{\vec{k}-}^{\omega_{-}}$. The term $\gamma_{\vec{k} \vec{k}^{\prime \prime}}^{\omega}\left(Z, Z^{\prime \prime}, \vec{Q}_{\|}, \Omega\right)$ represents the irreducible vertex, which is physically interpreted here as the coherent light matter interaction in disordered granular non-linear systems. The Ward identity is derived in the generalized form for the scattering of photons in non-conserving media. Absorption or gain yield an additional contribution, and a form of the Ward-Takahashi identity for photons in complex matter $[40,43,44]$ is derived. Effectively the additional contribution is not negligible and thus presents in the theoretical results of the transport characteristics of the self-consistent framework. It also renormalizes the energy transport velocity $[31,34,40]$. The solution to Equation (6), is derived as the energy density response $\Phi_{\epsilon \epsilon}$ in the form of a diffusion pole, Equation (7)

$$
\Phi_{\epsilon \epsilon}=\frac{N_{\omega}}{\Omega+i D Q_{\|}^{2}+i D \xi^{-2}} .
$$

$$
\begin{aligned}
& {\left[2 \operatorname{Re}\left(\epsilon_{b}\right) \omega \Omega\right.}\left.-2 \vec{k}_{\|} \cdot \vec{Q}_{\|}+2 i k_{z} \partial_{Z}+\Delta \Sigma-\Delta \epsilon_{b} \omega^{2}\right] \Phi_{\vec{k} \vec{k}^{\prime}}^{\omega}\left(Z, Z^{\prime}, \vec{Q}_{\|,}, \Omega\right) \\
&=\Delta G \delta\left(\vec{k}-\vec{k}^{\prime}\right)+\sum_{Z^{\prime \prime}} \Delta G \int \frac{\mathrm{d}^{3} k^{\prime \prime}}{(2 \pi)^{3}} \gamma_{\vec{k} \vec{k}^{\prime \prime}}^{\omega}\left(Z, Z^{\prime \prime}, \vec{Q}_{\|}, \Omega\right) \Phi_{\vec{k}^{\prime \prime}}^{\omega} \vec{k}^{\prime} \\
&
\end{aligned}
$$

We define the correlation length $\xi$ in dependency to the energy density $\Phi_{\epsilon \epsilon}$ itself

$$
\frac{1}{\xi^{2}(Z)}=\frac{N_{\omega}}{D(\Omega=0 ; Z) \Phi_{\epsilon \epsilon}\left(Q_{\|}=0, Z ; \Omega=0\right)} .
$$


The full diffusion coefficient $D(\Omega=0 ; Z)$ is derived as

$$
D(\Omega)=D_{0}^{\text {tot }}-\tau^{2} D(\Omega) M(\Omega)
$$

where the bare diffusion $D_{0}=\frac{2 v_{E} c_{p}}{\omega \Delta G} \int \frac{d^{3} k}{(2 \pi)^{3}}(\vec{k} \cdot \hat{Q})^{2} \Delta G$ is complemented by the contributions originating from the active medium as the scatterers $D_{s}=\frac{1}{8} r_{\epsilon} A_{\epsilon} \tau^{2} \tilde{D}_{0}$ or the background $D_{b}=$ $\frac{1}{4}(\omega \tau)^{2} \Delta \epsilon_{b} \tilde{D}_{0}$. Thus the diffusion coefficient without memory effects reads $D_{0}^{\text {tot }}=D_{0}+D_{s}+D_{b}$. The term $-\tau^{2} D(\Omega) M(\Omega)$ contains the memory kernel $M(\Omega)$, Equation (9), including the Cooperon contribution and consequentially all interferences. $\tilde{D}_{0}$ equals $D_{0}$ where the imaginary part $\Delta G$ is replaced by the real part $\square G$. The renormalized density of states $N_{\omega}$ is derived as follows

$$
N_{\omega}=\frac{\omega^{2} \Delta G_{0}(\vec{Q}, \Omega)}{c_{p}^{2} g_{\omega}^{(1)}[1+\Delta(\omega)]} .
$$

We further use the notation and the abbreviations of [34]. $\Delta(\omega)=B_{\epsilon} A_{\epsilon}+i r_{\epsilon} \partial_{\Omega} A_{\epsilon}(\Omega)$ with $A_{\epsilon}=$ $2\left[u_{\epsilon} \operatorname{Re} G_{o}+\operatorname{Re} \Sigma_{o}\right]$ and $B_{\epsilon}=\frac{(\operatorname{Re} \Delta \epsilon)^{2}+(\operatorname{Im} \Delta \epsilon)^{2}}{2 \omega^{2}(\operatorname{Re} \Delta \epsilon)^{2}}, \partial_{\Omega}$ is the differential resulting from the expansion in $\vec{Q}$ and $\Omega$, where the momentum integrated form of $\Delta G$ is $\Delta G_{0}=\int \frac{\mathrm{d}^{3} k}{(2 \pi)^{3}} \Delta G_{p}^{\omega}(Q, \Omega, Z)$ and $\Delta \epsilon=\epsilon_{s}-\epsilon_{b}$, $u_{\epsilon}=\frac{\operatorname{Im}\left(\Delta \epsilon \Sigma^{\omega}\right)}{\operatorname{Im}\left(\Delta \epsilon G_{0}^{\omega}\right)}$ and the abbreviations $r_{\epsilon}=\operatorname{Im} \Delta \epsilon / \operatorname{Re} \Delta \epsilon$ and $g_{\omega}^{(1)}=\frac{4 \omega}{c^{2}} \operatorname{Re} \epsilon_{b}$, as well as $g_{\omega}^{(0)}=\frac{2 \omega}{c^{2}} \operatorname{Im} \epsilon_{b}$ are introduced.

The energy transport velocity is derived as $v_{E}=\frac{c^{2}}{c_{p} \operatorname{Re} \epsilon_{b}} \frac{1}{1+\Delta(\omega)}$, whereas $c_{P}=\operatorname{Re} \frac{c}{\sqrt{\epsilon_{b}-\Sigma_{0}^{\omega} \frac{c^{2}}{\omega^{2}}}}$ equals the phase velocity, each one is self-consistently derived. A detailed discussion of the time scale $\tau$ is found in reference [34]. By solving the renormalized diffusion equation Equation (13)

$$
i D \xi^{-2}=-i D \chi_{d}^{-2}-c_{1}\left(\partial_{Y}^{2} \Phi_{\epsilon \epsilon}(Q, \Omega)\right)+c_{2} .
$$

coupled with the energy density to the 4-level laser rate equations, see Section 2.3, the coefficients $c_{1}$ and $c_{2}$ are self-consistently dereived, and we arrive the spatial distribution of energy density

$$
-\frac{\partial^{2}}{\partial Y^{2}} \Phi_{\epsilon \epsilon}=\frac{1}{D} \frac{D}{-\chi_{d}^{2}} \Phi_{\epsilon \epsilon}+\text { ASE. }
$$

The term for nonlinear self-consistent microscopic random laser gain $\gamma_{21} n_{2}$, see Section 2.3, incorporates the influence of the boundary renormalized length scale $\chi_{d} ; \gamma_{21}$ is the transition rate from laser level 2 to level $1, n_{2}$ is the electronic occupation number of level 2 . This yields the inversion condition

$$
\frac{D}{-\chi_{d}^{2}}=\gamma_{21} n_{2}
$$

in stationary state. $\chi_{d}$ is the length scale implicated by dissipation in the bulk alone. The modification of the boundary, Equation (13), specifies the relation between lasing emission and amplified spontaneous contributions (ASE), Equation (14).

\subsection{Coupling to the Four Level Laser Rate Equations for Quantum Cascades}

The lasing dynamics in our theoretical framework are included by a four-level laser rate equation system, which is well known from quantum cascade lasers $[35,36,45]$ 


$$
\begin{aligned}
\frac{\partial N_{3}}{\partial t} & =\frac{N_{0}}{\tau_{P}}-\frac{N_{3}}{\tau_{32}} \\
\frac{\partial N_{2}}{\partial t} & =\frac{N_{3}}{\tau_{32}}-\left(\frac{1}{\tau_{21}}+\frac{1}{\tau_{n r}}\right) N_{2}-\frac{\left(N_{2}-N_{1}\right)}{\tau_{21}} n_{p h} \\
\frac{\partial N_{1}}{\partial t} & =\left(\frac{1}{\tau_{21}}+\frac{1}{\tau_{n r}}\right) N_{2}+\frac{\left(N_{2}-N_{1}\right)}{\tau_{21}} n_{p h}-\frac{N_{1}}{\tau_{10}} \\
\frac{\partial N_{0}}{\partial t} & =\frac{N_{1}}{\tau_{10}}-\frac{N_{0}}{\tau_{P}} \\
N_{\text {tot }} & =N_{0}+N_{1}+N_{2}+N_{3},
\end{aligned}
$$

All transition times $\tau_{i}$ here are given as the inverse of the transition rates $\gamma_{i}$, see for details Figure $1 b, \gamma=1 / \tau_{i}$. The numbers represent the laser levels. $\gamma_{21}=1 / \tau_{21}$ represents the transition from upper to lower laser level, $\gamma_{P}$ is the pump rate, $\gamma_{n r}$ represents nonradiative decay. While we included nonradiative decay processes in previous work [36] for polydisperse $\mathrm{ZnO}$ powders, they are neglected in this work here. The numbers $N_{i}$ represent the level occupation and their resummation $N_{\text {tot }}$ ensures energy as well as particle conservation. The number $n_{p h}$ represents the photon numbers due to spontaneous emission and due to lasing. The coupling to the transport theory is given by the stimulated decay procedures, i.e., by Equation (18), which is connected to Equation (15). In due course of this article the transition rate $\gamma_{21}$ serves as the measure of the pump strength $P$.

\section{Results and Discussion}

\subsection{The Coherence Volume of $D=3$ Dimensional Random Lasers}

We find a full set of self-consistent scales, e.g., the diffusion coefficient $D$, the scattering mean-free path $l_{s}$, the transport mean-free path or coherence length $\xi$, the energy transport velocity $v_{E}$, the phase velocity $c_{p}$, the energy and intensity dependent LDOS $N_{\omega}$, as well as the laser thresholds and the random laser gain in stationary state from the solution of the Bethe-Salpeter equation and the intensity correlator including interference effects $\Phi_{\epsilon \epsilon}$.

As the main solution from the generalized diffusion equation, Equation (13), we derive the coherence length $\xi$ in a 3-dimensional setup with one finite dimension $z$. The finite system is characterized by lossy boundaries, otherwise we neglect losses for our considerations here in order to derive a systematic presentation of the random lasing phase transition. The translation invariance is consequentially broken in the $z$ direction. We present in Figure 2 results for one specific set of a possible random laser sample under three different excitation regimes with respect to the microscopic bulk matter properties of the scatterer's material, sub-threshold excitation with respect to bulk, excitation at the bulk threshold and excitation above the bulk threshold.

We show results for an arrangement of diamond nano resonators of refractive index $n=2.4$ with the radius of the single scatterer $r_{\text {scat }}=423.0 \mathrm{~nm}$ in a slab geometry of $50 \%$ filling and the extent of $80 r_{\text {scat }}=33.84 \mu \mathrm{m}$ in z-direction whereas it is infinite in-plane. The sample is equally lossy on either of the boundaries. We assume spatially homogeneous pumping of the sample and we show the results for the excitation strengths $P=0.2 \gamma_{21}, P=0.5 \gamma_{21}, P=1.0 \gamma_{21}$ and $P=9.0 \gamma_{21}$, where $\gamma_{21}$ is the rate for stimulated emission at the lasing transition of the bulk matter alone. We thus focus on four possible regimes of the bulk reference and we check whether the same classification still holds for the disordered arrangements where we believe that multiple scattering between otherwise independent active nano resonators will be the leading order effect in solid state random laser samples of a high filling fraction. 


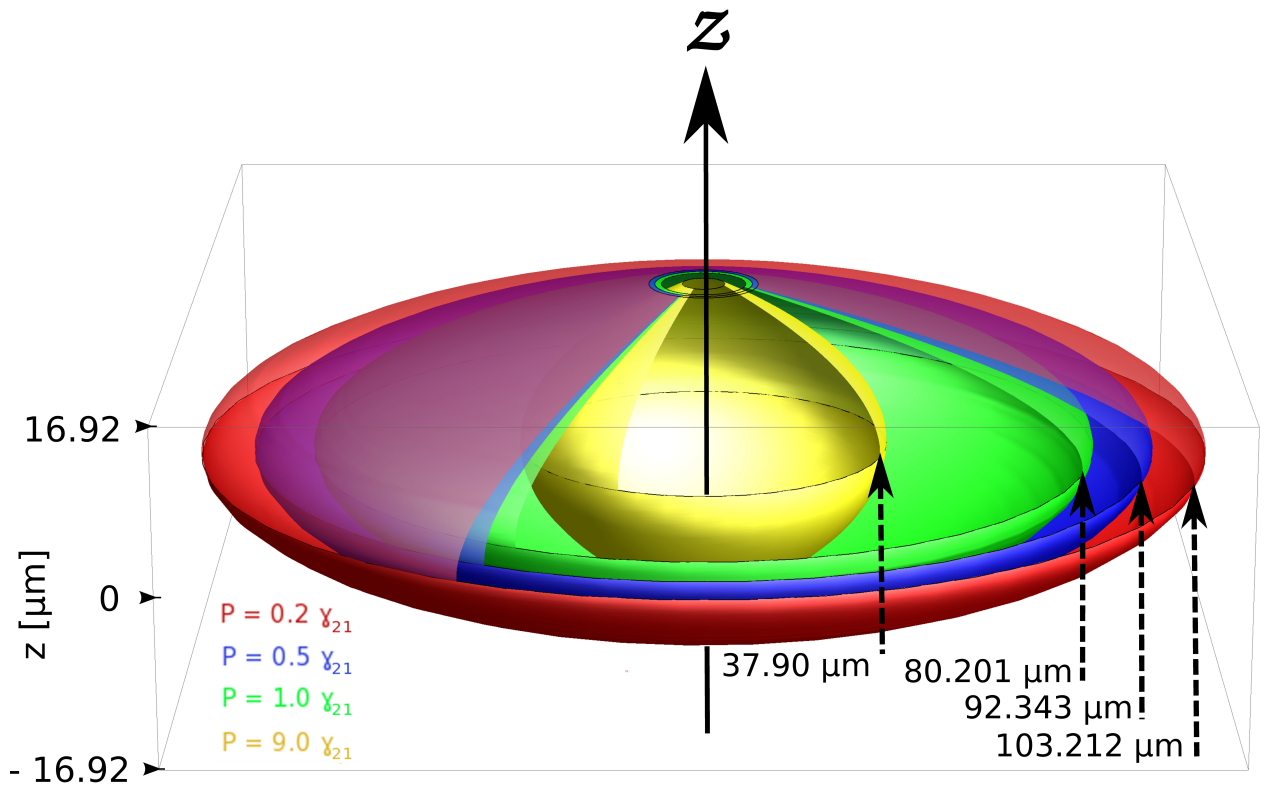

Figure 2. Coherence volume as the solution from Equation (10) within a $\mathrm{D}=3$ dimensional random laser slab of monodisperse Mie scatterers. The scatterers' radius is $r_{\text {scat }}=423.0 \mathrm{~nm}$, the particles refractive index is $n=2.4$, the real part of permittivity is $\operatorname{Re} \epsilon_{\text {scat }}=5.76$, which is comparable to diamond (C). The transport wavelength for this result is chosen as $\lambda=385 \mathrm{~nm}$, the samples filling fraction is chosen as $50 \%$ volume filling. The sample is of finite size in the z-direction, its extension in z-direction is $d=80 r_{\text {scat }}=33.84 \mu \mathrm{m}$. The strongly scattering medium is thus open, and the translation invariance is broken in the z-direction, whereas the sample is infinite in the $x-y$ plane. We show results for the excitation rates of $P=0.2 \gamma_{21}$ (red), $0.5 \gamma_{21}$ (blue), $1.0 \gamma_{21}$ (green), $9.0 \gamma_{21}$ (yellow) in units of the stimulated emission rate $\left[\gamma_{21}\right]$, see Figure $1 b$. The stationary state coherence volume for each value of the excitation power assumes in stationary state the form of a three dimensional extended disk, which is symmetrical in plane of the slab. The radius of the disk for each excitation power is noted in the figure. The disk is a spatial measure for the random laser threshold of the specific random laser arrangement. At the samples boundaries, the coherence length assumes a finite value which depends on the pump strength. It shall be noted that in cases for $P<1.0 \gamma_{21}$ the external excitation strength is smaller than the threshold value for bulk matter. Thus in this regime the multiple scattering procedures between Mie resonators are the dominating physical concept for the lasing transition of the random scatterer arrangement.

As a result we find that large scale systems can be driven with subcritical excitation strengths up to the laser transition. Here we show the case for $P=0.2 \gamma_{21} \ldots P=0.5 \gamma_{21}$. The pump power is definitely far below the microscopic laser threshold and thus is subcritical. For $P=0.2 \gamma_{21}$ we obtain a symmetrically shaped coherence volume with respect to the z-axis with a radius in the sample's center, $Z=0$, of $\xi=103.212 \mu \mathrm{m}$, see Figure 2 . Thus the coherence volume has an extent in the sample's center of $2 \cdot \xi=206.424 \mu \mathrm{m}$. By increasing the pump rate to $P=0.5 \gamma_{21}$ the coherence length reduces quantitatively to $\xi=92.343 \mu \mathrm{m}$. At the threshold value of the bulk material $P=1.0 \gamma_{21}$ we find qualitatively the same behavior; however, the quantity is reduced again to $\xi=80.201 \mu \mathrm{m}$. By increasing the pump power to $P=0.5 \gamma_{21}$ we find the coherence length of $\xi=37.90 \mu \mathrm{m}$. The length scale $\xi$ is a direct measure of the random laser mode in stationary state under specific conditions. It is also a measure of the Cooperon contribution, which is the perfect interference of a photon and its time reversal in multiple scattering procedures that acts as the stimulation process 
in a random laser. The coherence volume is thus a measure of the correlation of multiple scattering events between otherwise independent scatterers. At the samples boundaries the coherence length $\xi$ becomes always finite. The coherence length $\xi$ is derived in the stationary state, which means that it is the quantitative length scale which is the measure for the system's random laser threshold. We find no crossover of these length scales $\xi$ for varying pump rates anywhere in the sample with otherwise identical parameters.

\subsection{Scattering mean-free Path and Diffusion Coefficient at the Random Laser Threshold}

We show in Figure 3a the result for the scattering mean-free path $l_{s}$ that is computed within the self-consistent framework in dependency to the pump strength $\mathrm{P}$ and the position inside the sample. We find a significant qualitative difference to the same length scale in the non-pumped regime. The length scale $l_{S}$ at the random laser threshold, thus in stationary state, is increased at the sample's lossy boundaries. This effect relies here exclusively on the incorporation of a loss rate at the boundaries, whereas the sample's filling is $50 \%$ volume filling everywhere in the slab. We find that the increase of $l_{s}$ is qualitatively not directly inverse to the behavior of the coherence or correlation length $\xi$; however it depends on the strength of the excitation power. This is intuitively clear, since with the increasing excitation power of the pump the refractive index of the single active Mie resonator is modulated. The sample is homogeneously pumped, all parameters are equal to the case of Figure 2. We also find a significant quantitative difference of the scattering mean-free path $l_{s}$ at the laser threshold, which is for $P=9.0 \gamma_{21}$ at the boundaries, $l_{s}=1460.0 \mathrm{~nm}$ and in the center of the slab, at $Z=0, l_{s}=1432.0 \mathrm{~nm}$. Thus $l_{s}$ under stationary state lasing conditions is increased for our parameter set here by a factor of 4.7 to a factor of 4.87 compared to the measure $l_{s}$ in the non-pumped system, whereas its quality in terms of the derived formula is exactly the same, compare reference [34].

Our results for $l_{s}$, Figure $3 \mathrm{a}$, and $D$, Figure $3 \mathrm{~b}$, are as such remarkable, since they provide some insight on the physical consequence of the pumping of complex active disordered media with respect to their localization characteristics. The measure for localization is always the self-consistent diffusion coefficient $D$, that we show as a result for the same parameters as for the calculation of $l_{s}$, Figure 3a, and for the calculation of $\xi$, Figure 2, in Figure $3 b$. The diffusion coefficient shows qualitatively the same behavior as $l_{s}$ in stationary state. It assumes quantitatively a finite value which depends on the position and on the pump strength. We thus conclude that random lasers at the stationary state undergo the lasing transition, which is, however, neither correlated nor equal to the phase transition of Anderson localization.

\subsection{Material-Dependency of the Mie Characteristics in Multiple Scattering Random Lasers}

We know from the results for the scattering mean-free path $l_{s}$ for photonic transport in the weak excitation regime that a crossover of $l_{s}$ is expected for various Mie resonators of a differing refractive index $n$ and thus a differing permittivity $\epsilon_{\text {scat }}$. The result for the calculation of the scattering mean-free path [34] shows smooth but pronounced dips when the Mie resonance condition for the single scatterer is approached and finally met. This Mie resonance condition depends on the refractive index of the scatterer; its position will thus shift in the energy spectrum for various materials and identical scatterer parameters otherwise. Under an ideal choice of parameters an almost perfect point symmetry with respect to the crossover of the scattering mean free path $l_{s}$ for two different refractive indices is possible.

Here we refer to the literature, references [1,2], where the gain mechanism in solid state random lasing is vividly discussed with a focus on two possible mechanisms, microscopic gain and gain due to artificial but randomly formed laser cavities in the sense of a build up of correlated chains of single scatterers. Whereas for the case of microscopic gain the properties of the single Mie scatterer should change with respect to the resulting change of the refractive index, in the case of artificial but randomly formed laser cavities the properties of the single scatterer should remain rather unchanged. 
(a) Scattering mean-free path $\mathrm{l}_{\mathrm{S}}$ in the laser active system at threshold

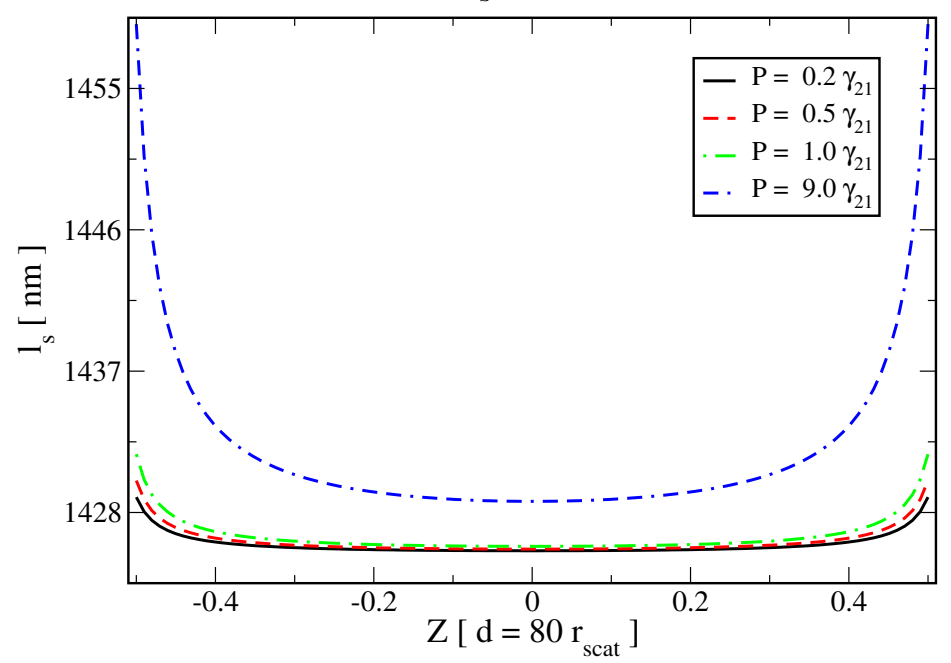

(b) Diffusion coefficient D of the random laser in stationary state

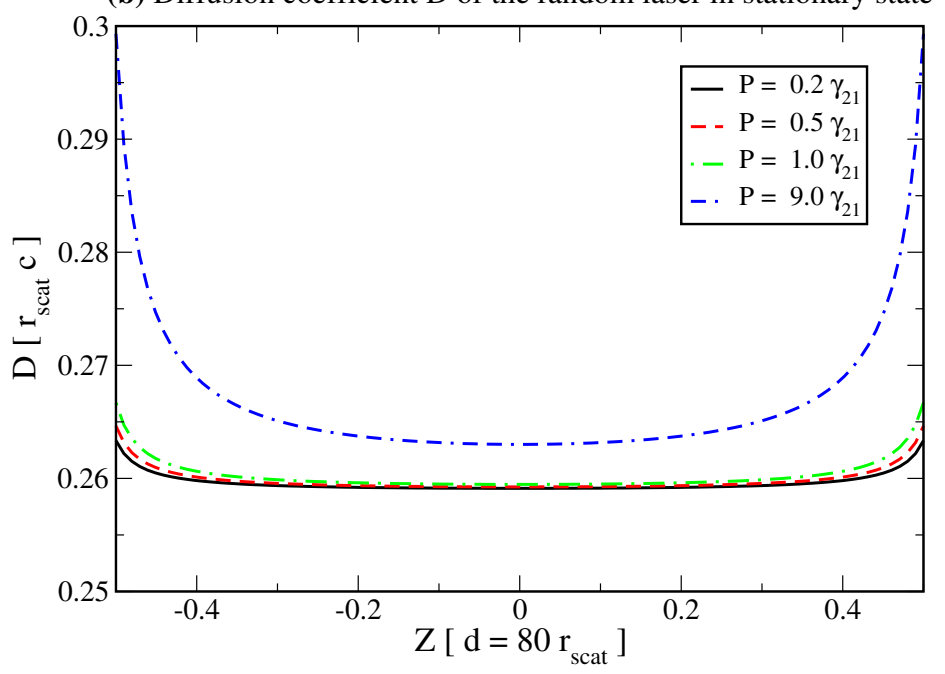

Figure 3. (a) Scattering mean-free path $l_{S}$ in the laser active system at threshold. In-depth dependent scattering mean-free path $l_{s}=\frac{1}{2 \operatorname{Im}\left[\sqrt{q^{2}+i \operatorname{Im} \Sigma(\omega)}\right]}$, for details see [34], in dependency to the excitation strength $P$. Parameters are identical to those of Figure 2 . We find an in-depth dependent renormalization of $l_{s}$ which is increased with strength $P$. Both quantities, the mean-free path $l_{s}$ and the diffusion coefficient $D(\Omega=0 ; Z)$, Figure $3 b$, are self-consistently derived and thus show quantitatively a different behavior at the sample boundaries $Z= \pm 0.5 d$, rather than at $Z=0$. At the open boundaries both characteristics $l_{S}$ and $D(\Omega=0 ; Z)$ are increased. This behavior comes, however, along with the huge but inverse effect in the characteristics of the coherence volume, compare Figure 2. (b) Diffusion coefficient $D$ of the random laser in stationary state. In-depth dependent diffusion coefficient $D(\Omega=0 ; Z)$ as a material characteristic of the random laser arrangement at the laser threshold in the stationary state. Parameters are identical to Figures 2 and 3a. In the excitation regime $P<1.0 \gamma_{21}$ a moderate but existing dependency with respect to the external excitation and the position in-depth is found. In the regime for excitation above the microscopic laser threshold $P<9.0 \gamma_{21}$ we find an increasing deviation of the value of the diffusion coefficient $\mathrm{D}$ at the sample boundary of more than $7 \%$ as compared to the transport wavelength. This deviation is only correlated to the absolute excitation power and to the open boundaries of the sample where $85 \%$ of all reemitted photons shall be lost, whereas the in-depth sample of all photons multiplies scattering. Both results of $l_{s}$ and of $D$, which is finite, are signs of the absence of Anderson localization [46]. 
This discussion for sure will be finally decided by novel experiments, which check the spatial extent of random laser modes in stationary state systematically by varying the geometric properties of the laser sample. So far we can deduce from our results that for solid state random lasers gain due to artificial but randomly formed laser cavities is not the leading order gain mechanism, since the refractive index is obviously changed and the scattering mean-free path in stationary state is increased in comparison to the same system under weak excitation, compare Section 3.2. We check this behavior theoretically in order to propose possible experiments which may be feasible to address the issue, and one of them is to compare the measurable result of the coherence volume to the crossover behavior of the scattering mean-free path for the same but only weakly excited, so non-pumped, system in a frequency range where we know the crossover of $l_{s}$ in the weakly excited case is very pronounced. For the system paremeters $r_{\text {scat }}=423.0 \mathrm{~nm}$, the samples extent in z-direction of $d=80 r_{\text {scat }}=33.84 \mu \mathrm{m}$, a filling fraction of $50 \%$ and the refractive indices of $n=2.4$ (C) and $n=2.0041(\mathrm{ZnO})$ such a very clear crossover of $l_{s}$ is found at $\lambda=600.0 \mathrm{~nm}$, compare Figure $4 \mathrm{~b}$. We show in Figure 4a the in-depth dependency of the correlation length $\xi$ for both systems, $\mathrm{C}$ and $\mathrm{ZnO}$, under moderate pumping $P=1.0 \gamma_{21}$. The pump wavelength as well as the transport wavelength are chosen as $\lambda=580.0 \mathrm{~nm}$ and $\lambda=620.0 \mathrm{~nm}$. We find two qualitatively different crossover situations. First we find an obvious crossover of $\xi$ for the parameters $n=2.4$ and $\lambda=580.0 \mathrm{~nm}$ in the outer selvedge of the sample, which is in the outer $10 \%$ of the samples extent on either open boundary. Second we find a qualitative change of the coherence length $\xi$ when we consider a different material by changing the passive refractive index from $n=2.0041$ to $n=2.4$. For the parameters $\lambda=580.0 \mathrm{~nm}$ and $n=2.0041$ the coherence length $\xi$ all over the sample depth obtains almost the same characteristics and the same value as $\xi$ for the case of $n=2.4$ and $\lambda=620.0 \mathrm{~nm}$. The direct counterparts, $\xi$ for the parameter set of $n=2.0041, \lambda=620.0 \mathrm{~nm}$, and on the other hand $\xi$ for the set of $n=2.4$ and $\lambda=580.0 \mathrm{~nm}$ show a significant difference of about $40 \%$. This crossover becomes more obvious, when we display the coherence lengths $\xi$, as single marked points, in comparison to the scattering mean-free path $l_{s}$ for the weakly excited regime, marked as the solid and the dashed line in Figure $4 \mathrm{~b}$. Whereas the absolute value of $l_{s}$ is rather symmetric for the refractive indices $n=2.0041$ and $n=2.4$ in the spectral positions of $\lambda=580.0 \mathrm{~nm}$, and of $\lambda=620.0 \mathrm{~nm}$, to their crossover at $\lambda=600.0 \mathrm{~nm}$, the coherence length by contrast shows a crossover as well, but the almost perfect point symmetry behavior of the scattering mean-free path in the weakly excited case is not present in the crossover behavior of $\xi$ in stationary state. The crossover of $\xi$ is also not confirmed for any crossover point of $l_{s}$ for weakly excited Mie resonators in the spectrum.

\subsection{Power Law Behavior of the Correlation Length Scale in Stationary State}

We have investigated the coherence length $\xi$ with increasing pump power for various refractive indices and various sample extents. In Figure 5 we show the coherence length $\xi$ for the scatterers arrangements of $r_{\text {scat }}=423.0 \mathrm{~nm}$, filling fraction of $50 \%$ and the samples extents of $d=40 r_{\text {scat }}=$ $16.92 \mu \mathrm{m}$ and $d=80 r_{\text {scat }}=33.84 \mu \mathrm{m}$. We show samples for $n=2.4(\mathrm{C}), n=2.3(\mathrm{ZnO})$ and $n=3.22\left(\mathrm{TiO}_{2}\right)$. The excitation wavelength is $\lambda=385.0 \mathrm{~nm}$; results are displayed for pump powers from $0.2 \gamma_{21}$ up to $9.0 \gamma_{21}$. We display the dependency of $\xi$ with $1 / \sqrt{P}$ and we find a characteristic power law behavior for increasing pump power $P$. Another, similar power law has been found in experiments of random lasers by Cao et al. [2]. When we compare several materials we find the specific crossover behavior for the coherence length $\xi$ for the samples of $n=2.4$ (C) and $n=3.22$ $\left(\mathrm{TiO}_{2}\right)$ whereas the power law behavior is confirmed in either sample. The coherence length $\xi$ for $\mathrm{TiO}_{2}$ assumes values of up to $\xi=150.0 \mu \mathrm{m}$; thus the coherence volume has an extent in this case of up to $\xi=300.0 \mu \mathrm{m}$ in stationary state. 
(a) Crossover of $\xi$ for varying refractive index $\mathrm{n}$ and wavelength $\lambda$

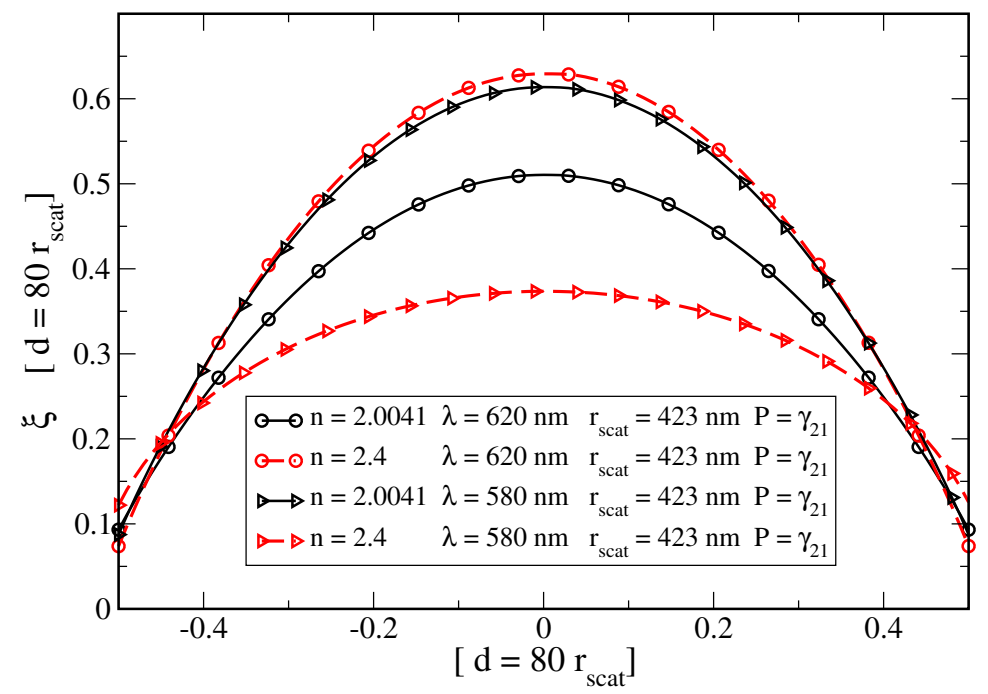

(b) Coherence length $\xi$ in stationary state and $\mathrm{l}_{\mathrm{S}}$ below threshold

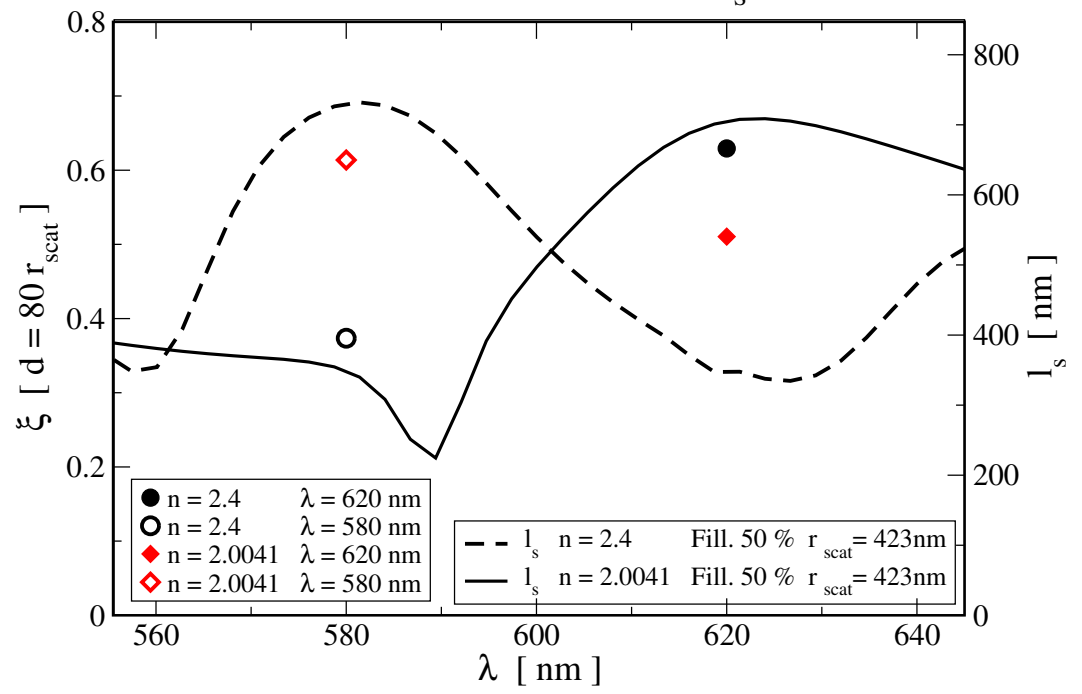

Figure 4. (a) Crossover of the coherence length $\xi$ for various refractive indices. The crossover of the coherence length $\xi$ for two materials $n=2.4(\mathrm{C})$ and $n=2.0041(\mathrm{ZnO})$ is displayed for the transport wavelengths $\lambda=580.0 \mathrm{~nm}$ and $\lambda=620.0 \mathrm{~nm}$ in-depth dependent of the random laser sample. The system parameters apart from the refractive index $n$ and the transport wavelength $\lambda$ are in either case identical, the scatterers radius is $r_{\text {scat }}=423.0 \mathrm{~nm}$. The samples finite extent in z-direction is of $80 r_{\text {scat }}$, the excitation strength $P=1.0 \gamma_{21}$ is moderate. A principal crossover behavior of the length scale $\xi$ is derived. We also find for the refractive index $n=2.4$ a crossover of the coherence lengths $\xi$ near the sample's boundaries for $\lambda=580.0 \mathrm{~nm}$ and $\lambda=620.0 \mathrm{~nm}$ in the stationary state. (b) Coherence length $\xi$ compared to the scattering mean-free path $l_{s}$ below threshold. The crossover of the coherence length $\xi$ is shown in the samples center, $Z=0$, parameters are identical to Figure $4 \mathrm{a}$. Intuitively a crossover behavior of $\xi$ is expected from the investigation of the scattering mean-free path $l_{s}, n=2.4(\mathrm{C})$ (dashed line), $n=2.0041$ ( $\mathrm{ZnO}$ ) (solid line), for disordered arrangements of Mie scatterers below the laser threshold $[31,34]$. Opposed to our results for $l_{s}$ at the stationary state, see Figure $3 \mathrm{a}$, we compare here to $l_{s}$ in the weakly pumped case. In the spectral region between $\lambda=580.0$ $\mathrm{nm}$ and $\lambda=620.0 \mathrm{~nm}$ we can confirm the crossover, which means that the Mie characteristics persist as a leading order effect under these specific conditions also while the system undergoes the lasing transition. 


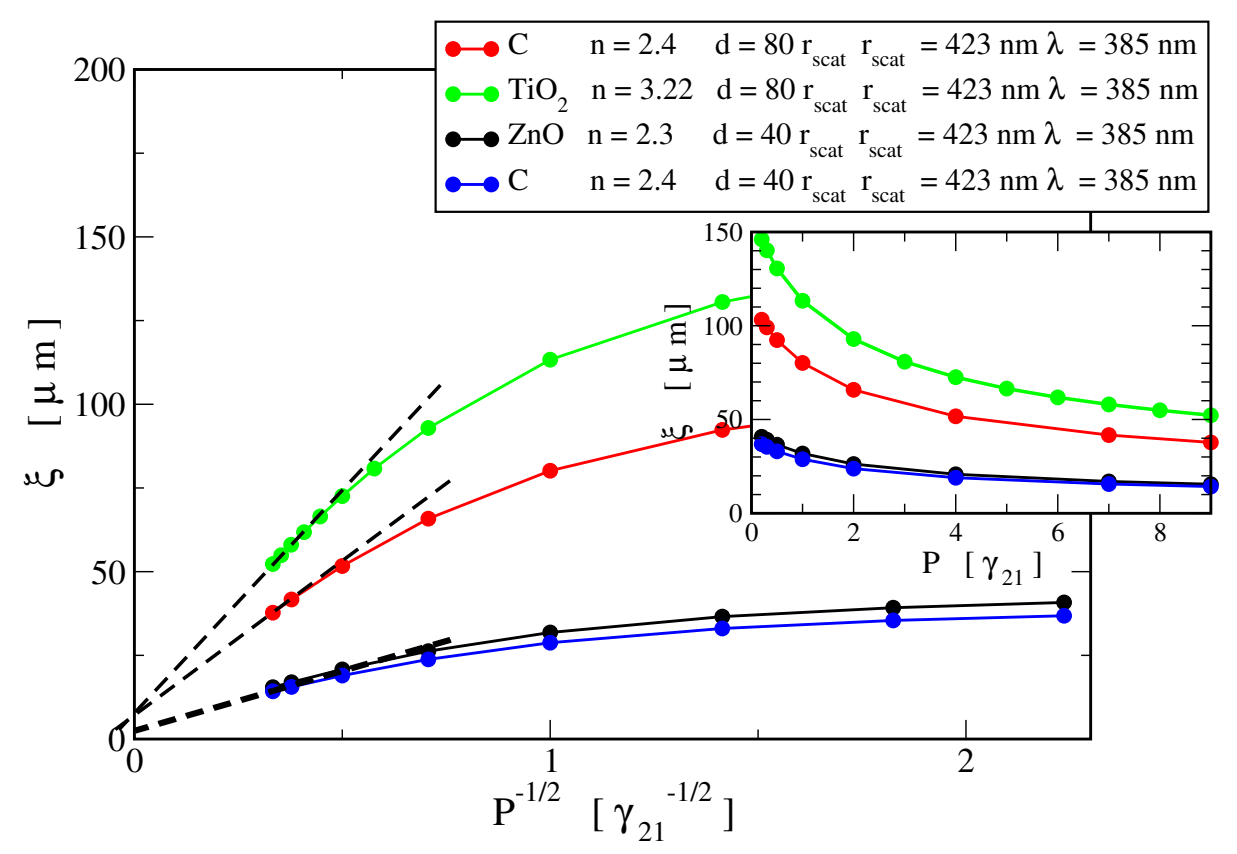

Figure 5. Self-consistent coherence length $\xi$ of the random laser system in the finite size slab arrangement with a filling fraction of $50 \%$ for four different setups. We show $\xi$ at the samples center $Z=0$ for various extents of the slab with its dependency to the excitation power. Parameters are $d=40 r_{\text {scat }}$ and $d=80 r_{\text {scat }}, r_{\text {scat }}=423.0 \mathrm{~nm}$. Results are shown for identical scatterers' radii of $\mathrm{ZnO}$ (zinkoxide, $n=2.3$ ), of $\mathrm{C}$ (diamond, $n=2.4$ ) and of $\mathrm{TiO}_{2}$ (titania, $n=3.22$ ) active Mie scatterers without any amplifying or absorbing background, $\epsilon_{b}=1$. . With increasing excitation power $P$ a transition to a power law behavior of $\xi$ with $1 / \sqrt{P}$ is found in either of the setups (see black dashed lines). At the transport wavelength $\lambda=385.0 \mathrm{~nm}$ we find that the Mie characteristic is a leading order effect in stationary state lasing. We find for the scatterers radius $r_{\text {scat }}=423.0 \mathrm{~nm}$ and $\lambda=385.0 \mathrm{~nm}$ for C, $n=2.4$, and titania, $n=3.22$, a significant difference in $\xi$ which cannot be explained by bulk effects. We attribute it to enhanced multiple scattering for the diamond Mie resonators with these parameters.

\section{Conclusions}

We have presented in this article a quantum field theoretical approach for three dimensional solid state random lasers comprised of active complex Mie resonators. Thus we have implemented the Ward identity for non-conserving media. The systems are open at the boundaries. As a result we derived the coherence volume of random lasers as the spatial characteristics of the random laser threshold in the stationary state. This includes the spatial dependency of the self-consistent laser gain. We conclude from our results that the random laser of densely packed strongly scattering random media is significantly depending on multiple scattering processes in the sense that stationary state lasing can be reached already for subcritical pump intensities, far below what is known as the microscopic laser threshold of the bulk material. This characteristics is also depending on Mie scattering characteristics of the active single scatterer. We have also presented the results for the diffusion coefficient and the scattering mean-free path which have been derived by the self-consistent framework for finite samples in the stationary lasing state and which are thus also spatially dependent. We find a significant deviation of the scattering mean-free path in the lasing regime in stationary state from $l_{s}$ the weakly excited case. This deviation is confirmed in the characteristics of the diffusion coefficient which is finite and spatially dependent. We compared the qualitative behavior of the coherence length of the stationary state random laser to the qualitative behavior of the scattering mean-free path of photons in the same but non-pumped and thus non-inverted setup. We find that the coherence length in stationary state qualitatively follows the material dependent spectral crossover behavior of the scattering mean-free path of disordered samples of weakly excited Mie resonators and otherwise identical parameters in the case of very pronounced Mie characteristics in weakly excited 
systems. We also compared the coherence length in stationary state for identical scatterers of various materials, diamond $(\mathrm{C})$, zinkoxide $(\mathrm{ZnO})$ and titania $\left(\mathrm{TiO}_{2}\right)$, for increasing pump strength as well as for varying samples thicknesses. We find in all our results a power law behavior of the coherence length with respect to the pump intensity. We conclude from our theory that for the understanding of the physics of random lasers of multiple scattering ensembles, the concept of the localization length which is a fundamental characteristics for mesoscopic transport of light in random media is conceptually not sufficient since it does not provide knowledge about the effect of self-consistent gain including the microscopic electronic subsystem. The length scale of the coherence or correlation length and the coherence or correlation volume, respectively, are more comprehensive scales for laser active random media and they characterize the laser modes and the laser threshold behavior. We investigated in this work arrangements of independent monodisperse Mie resonators. It will be the subject of future work to investigate the influence of gain and absorption with respect to sub- and hyper-diffusion systems and with respect to laser samples of quasi-ordered clusters and meta glasses.

Author Contributions: All authors contributed equally to this work. All authors wrote and reviewed the manuscript. Funding: This research received no external funding.

Acknowledgments: We thank H. Cao, B. A. van Tiggelen, K. Busch and J. Kroha for very fruitful discussions.

Conflicts of Interest: The authors declare no conflict of interest.

\section{References}

1. Cao, H.; Zhao, Y.G.; Ho, S.T.; Seelig, E.W.; Wang, Q.H.; Chang, R.P.H. Random laser action in semiconductor powder. Phys. Rev. Lett. 1999, 82, 2278-2281. [CrossRef]

2. Cao, H. Lasing in random media. Waves Random Media 2003, 13, R1-R39. [CrossRef]

3. Dice, G.D.; Mujumdar, S.; Elezzabi, A.Y. Plasmonically enhanced diffusive and subdiffusive metal nanoparticle-dye random laser. Appl. Phys. Lett. 2005, 86, 131105. [CrossRef]

4. Wiersma, D. The physics and applications of random lasers. Nat. Phys. 2008, 4, 359-367. [CrossRef]

5. Noginov, M.A.; Zhu, G.; Belgrave, A.M.; Bakker, R.; Shalaev, V.M.; Narimanov, E.E.; Stout, S.; Herz, E.; Suteewong, T.; Wiesner, U. Demonstration of a spaser-based nanolaser. Nature 2009, 460, 1110-1112. [CrossRef] [PubMed]

6. Polson, R.C.; Vardeny, Z.V. Spatially mapping random lasing cavities. Opt. Lett. 2010, 35, 2801. [CrossRef] [PubMed]

7. Tiwari, A.K.; Mujumdar, S. Random Lasing over Gap States from a Quasi-One-Dimensional Amplifying Periodic-on-Average Random Superlattice. Phys. Rev. Lett. 2013, 111, 233903. [CrossRef]

8. Uppu, R.; Mujumdar, S. Exponentially Tempered Lévy Sums in Random Lasers. Phys. Rev. Lett. 2015, 114, 183903. [CrossRef]

9. Niyuki, R.; Fujiwara, H.; Nakamura, T.; Ishikawa, Y.; Koshizaki, N.; Tsuji, T.; Sasaki, K. Double threshold behavior in a resonance-controlled $\mathrm{ZnO}$ random laser. APL Photonics 2014, 2, 036101. [CrossRef]

10. Shi, X.; Liao, Y.-M.; Lin, H.-Y.; Tsao, P.-W.; Wu, M.-J.; Lin, S.-Y.; Hu, H.-H.; Wang, Z.; Lin, T.-Y.; Lai, Y.-C.; et al. Dissolvable and Recyclable Random Lasers. ACS Nano 2017, 11, 7600-7607. [CrossRef]

11. Safdas, A.; Wang, Y.; Krauss, T.F. Random lasing in uniform perovskite thin films. Opt. Express 2018, 26, A75-A80. [CrossRef] [PubMed]

12. Lau, S.-P.; Yang, H.; Yu, S.F.; Yuen, C.; Leong, E.S.P.; Li, H.; Hng, H.H. Flexible Ultraviolet Random Lasers Based on Nanoparticles. Small 2005, 1, 956-959. [CrossRef] [PubMed]

13. Vasileva, E.L.; Li, Y.U.; Sychugov, I.; Mensied, M.; Berglund, L; Popov, S. Lasing from organic dye molecules embedded in transparent wood. Adv. Opt. Mater. 2017, 5, 1700057. [CrossRef]

14. Oivurova, M.A.K.; Vasileva, E.L.; Li, Y.U.; Berglund, L.; Popov, S. Complete spatial coherence characterization of quasi-random laser emission from dye doped transparent wood. Opt. Express 2018, 26, 13476.

15. Hackenbroich, G.; Viviescas, C.; Haake, F. Field quantization for chaotic resonators with overlapping modes. Phys. Rev. Lett. 2002, 89, 083902. [CrossRef] [PubMed]

16. Mujumdar, S.; Ricci, M.; Torre, R.; Wiersma, D.S. Amplified Extended Modes in Random Lasers. Phys. Rev. Lett. 2004, 93, 053903. [CrossRef] 
17. Hackenbroich, G. Statistical theory of multimode random lasers. J. Phys. A Math. Gen. 2005, 38, 10537. [CrossRef]

18. Mujumdar, D.; Türck, V.; Torre, R.; Wiersma, D.S. Chaotic behavior of a random laser with static disorder. Phys. Rev. A 2007, 76, 033807. [CrossRef]

19. Lepri, S. Fluctuations in a Diffusive Medium with Gain. Phys. Rev. Lett. 2013, 110, 230603. [CrossRef]

20. Höfner, W.; Wünsche, H.-J.; Henneberger, F. A random laser as a dynamical network. New J. Phys. 2014, 16, 033002. [CrossRef]

21. Lepri, S.; Trono, C.; Giacomelli, G. Complex Active Optical Networks as a New Laser Concept. Phys. Rev. Lett. 2017, 118, 12390. [CrossRef]

22. Gaio, M.; Saxena, D.; Bertolotti, J.; Pisignano, D.; Camposeo, A.; Sapienza, R. A nanophotonic laser on a graph. Nat. Commun. 2019, 10, 226. [CrossRef] [PubMed]

23. Jiang, X; Soukoulis, C.M. Time Dependent Theory for Random Laser. Phys. Rev. Lett. 2002, 85, 70-73. [CrossRef] [PubMed]

24. Soukoulis, C.; M.; Jiang, X.; Xu, J.Y.; Cao, H. Dynamic response and relaxation oscillations in random lasers. Phys. Rev. B 2002, 65, 041103R. [CrossRef]

25. Türeci, H.E.; Stone, A.D.; Collier, B. Self-consistent multi-mode lasing theory for complex or random lasing media. Phys. Rev. A 2006, 74, 043822. [CrossRef]

26. Türeci, H.E.; Ge, L.; Rotter, S.; Stone, A.D. Strong Interactions in Multimode Random Lasers. Science 2008, 320, 643-646. [CrossRef] [PubMed]

27. Conti, C.; Fratalocchi, A. Dynamic light diffusion, Anderson localization and lasing in disordered inverted opals: 3D ab-initio Maxwell-Bloch computation. Nat. Phys. 2008, 4, 794-798. [CrossRef]

28. Versteegh, M.A.M.; Vanmaekelbergh, D.; Dijkhuis, J.I. Room-Temperature Laser Emission of ZnO Nanowires Explained by Many-Body Theory. Phys. Rev. Lett. 2012, 108, 157402. [CrossRef]

29. Versteegh, M.A.M.; van Capel, P.J.S.; Dijkhuis, J.I. Ultrafast all-optical gated amplifier based on ZnO nanowire lasing. Appl. Phys. Lett. 2012, 101, 021101. [CrossRef]

30. Liu, J.; Garcia, P.D.; Gregersen, S.; Ek, N.; Suhr, T.; Schubert, M.; Mork, J.; Stobbe, S.; Lodahl, P. Random nanolasing in the Anderson localized regime. Nat. Nanotechnol. 2014, 9, 285-289. [CrossRef]

31. Frank, R.; Lubatsch, A.; Kroha, J. Theory of strong localization effects in disordered loss or gain media. Phys. Rev. B 2006, 73, 245107. [CrossRef]

32. Frank, R.; Lubatsch, A.; Kroha, J. Light transport and localization in diffusive random lasers. J. Opt. A Pure Appl. Opt. 2011, 11, 114012. [CrossRef]

33. Frank, R.; Lubatsch, A.; Kroha, J. Light transport and localization in diffusive random lasers. Ann. Der Phys. 2009, 18, 882-886. [CrossRef]

34. Frank, R.; Lubatsch, A. Scalar wave propagation in random amplifying media: Influence of localization effects on length and time scales and threshold behavior. Phys. Rev. A 2011, 84, 013814. [CrossRef]

35. Lubatsch, A.; Frank, R. Coherent transport and symmetry breaking-Laser dynamics of constrained granular matter. New J. Phys. 2014, 16, 083043. [CrossRef]

36. Lubatsch, A.; Frank, R. Tuning the Quantum Efficiency of Random Lasers-Intrinsic Stokes-Shift and Gain. Sci. Rep. 2015, 5, 17000. [CrossRef]

37. Gottardo, S.; Spienza, R.; Garcia, P.D.; Blanco, A.; Wiersma, D.S.; Lopez, C. Resonance-driven random lasing. Nat. Photonics 2008, 2, 429-432. [CrossRef]

38. Consoli, A.; Lopez, C. Decoupling gain and feedback in coherent random lasers: Experiments and simulations. Sci. Rep. 2015, 5, 16848. [CrossRef]

39. Vollhardt, D.; Wölfle, P. Diagrammatic, self-consistent treatment of the Anderson localization problem in $d<=2$ dimensions. Phys. Rev. B 1980, 22, 4666.

40. Lubatsch, A.; Kroha, J.; Busch, K. Theory of light diffusion in disordered media with linear absorption or gain. Phys. Rev. B 2005, 71, 184201. [CrossRef]

41. Mie, G. Beiträge zur Optik trüber Medien, speziell kolloidaler Metallösungen. Ann. Der Phys. 1908, 25, 377-445. [CrossRef]

42. Onsager, L. Reciprocal Relations in Irreversible Processes. I + II. Phys. Rev. 1931, 37-28, 405-426, 2265. [CrossRef]

43. Ward, J.C. An Identity in Quantum Electrodynamics. Phys. Rev. 1950, 78, 182. [CrossRef]

44. Takahashi, Y. On the Generalized Ward Identity. Il Nuovo Cimento 1957, VI, 2231-2235. [CrossRef] 
45. Faist, J.; Capasso, F.; Sivco, D.L.; Sirtori, C.; Hutchinson, A.L.; Cho, A.Y. Quantum Cascade Laser. Science 1994, 264, 553-556. [CrossRef] [PubMed]

46. Maret, G.; Sperling, T.; Buehrer, W.; Lubatsch, A.; Frank, R.; Aegerter, C.M. Reply to comment by F. Scheffold and D. Wiersma: Inelastic scattering puts in question recent claims of Anderson localization of light. Nat. Photonics 2013, 7, 934-935. [CrossRef]

(C) 2019 by the authors. Licensee MDPI, Basel, Switzerland. This article is an open access article distributed under the terms and conditions of the Creative Commons Attribution (CC BY) license (http:/ / creativecommons.org/licenses/by/4.0/). 